\title{
Refractive Outcome of Combined Phacoemulsification and Endoscopic Cyclophotocoagulation
}

\section{Authors}

Kang $\mathrm{S}^{1}$, Luk $\mathrm{S}^{1}$, Han $\mathrm{H}^{1}$, Cordeiro $\mathrm{MF}^{1,2,3}$, Ahmed $\mathrm{F}^{1,2}$, Bloom $\mathrm{P}^{1,2}$, Crawley $\mathrm{L}^{1,2}$

\section{Institution}

${ }^{1}$ Imperial College Healthcare NHS Trust

${ }^{2}$ Imperial College London

${ }^{3}$ Glaucoma \& Retinal Neuro-degeneration Research Group, UCL Institute of Ophthalmology, London

\section{Corresponding author}

Miss Swan Kang

Email: swan_kang@hotmail.com

Address: The Western Eye Hospital, 153-173 Marylebone Road, London NW1 5QH

Mobile: 07958585904

Phone: 02033126666

Fax: 02078866200

Keywords: phacoemulsification, endoscopic cyclophotocoagulation, phaco-ECP, refractive outcome, glaucoma cataract 
Financial Support: nil

The authors have no financial or proprietary interests in any material or method mentioned.

There is no involvement of research funding.

\section{Conflict of interests}

The authors declare no conflict of interests.

The findings has only been presented at the local hospital meeting - West London Research Medal 


\begin{abstract}
Purpose

To compare the refractive outcome of eyes that underwent phacoemulsification combined with endoscopic cyclophotocoagulation (phaco-ECP) with the eyes that underwent phacoemulsification alone.
\end{abstract}

Methods

A retrospective case series review of 103 consecutive eyes that underwent phaco-ECP and 62 eyes that underwent phacoemulsification between January 2006 and August 2012 was conducted. Post-operative refractive outcomes were obtained by subjective refraction or autorefraction. Demographic data, best corrected visual acuity (BCVA), intraocular pressure (IOP), number of glaucoma agents and mean deviation of the Humphrey visual field test (MD) were also analysed.

Results

The total number of eyes included in the study was 62 eyes for the phaco-ECP group and 62 eyes for the control group who underwent phacoemulsification alone. More than $90 \%$ of eyes $(n=56)$ in phaco-ECP group achieved post-operative refraction within +/- 1 dioptre (D) of the target refraction. In the control group $100 \%$ of eyes achieved post-operative refraction within $+/-1 \mathrm{D}$ of the target refraction. When comparing the difference between the target and the actual refractive outcome between the phaco-ECP and the control group, there was no statistically significant difference. 
Conclusions

Refractive outcome after phaco-ECP is comparable to phacoemulsification alone. This study suggests that the intraocular lens power can be selected as for cataract surgery alone and that ECP does not change the effective lens position significantly, therefore no modification of biometry formulae is required. Phaco-ECP should be considered as an effective, safe and predictable surgical treatment option for glaucoma patients with co-existing cataract. 


\section{Introduction}

Patients with glaucoma frequently develop cataract due to a number of plausible reasons such as the use of topical medications and previous laser or surgical procedures as well as physiological aging. ${ }^{1}$ Cataract itself may create challenges in glaucoma management by reducing vision, obstructing aqueous outflow and by making perimetry and optic disc assessment more difficult. Therefore, the timely management of both conditions is codependent in improving outcome.

Endoscopic cyclophotocoagulation (ECP) using a diode laser guided by an endoscope provides targeted ablation of the ciliary body under direct visualisation, while minimising surrounding tissue damage. ECP is gaining its popularity in the UK with currently 14 centres using the technology. The technique of combining phacoemulsification with endoscopic cyclophotocoagulation (phaco-ECP) has emerged as a viable option to simultaneously treat both cataract and glaucoma. ${ }^{2}$ The inherent advantages of the combined procedure include a single recovery period and effective reduction in IOP. ${ }^{3}$

Cataract surgery can correct refractive error by accurate selection of appropriate intraocular lens (IOL) power. ${ }^{4}$ As ECP causes contraction of the ciliary processes and the zonules insert on these processes, it may be hypothesised that this would change the effective position of the IOL which might in turn alter the postoperative refractive outcome adversely. The aim of this study was to investigate the refractive outcome of the combined phaco-ECP. 


\section{Materials and Methods}

Patients

The case records of 103 consecutive eyes that underwent phaco-ECP and 62 eyes of age-matched glaucoma patients who underwent phacoemulsification alone at a single tertiary referral center (The Western Eye Hospital, London, UK) between January 2006 and August 2012 were reviewed retrospectively. Patients with open angle glaucoma were included in the study, including normal tension, pseudoexfoliation, and pigmentary glaucoma subtypes. Patients with complete demographic, operative and follow-up data were subsequently entered into the study. Excluded from the study were patients referred from other centres for phaco-ECP procedures and subsequently had follow-up appointments at their local clinics and those who had visual acuities of counting fingers or worse or who were lost to follow-up. Pre- and post-operative Snellen visual acuities (VA) were recorded. Refraction was obtained by autorefraction performed at clinic visits (Canon Auto ref-keratometer RK5) or subjective refraction reported by optometrists. All complications were noted. Intraocular pressure (IOP) measured by calibrated Goldmann applanation tonometry, mean deviation (MD) of automated Humphrey visual field (SITA standard 24-2) and numbers of topical or systemic anti-glaucoma agents were also recorded.

Standard of Care

The Royal College of Ophthalmologists' cataract surgery guidelines 2010 set a benchmark for 85\% of patients undergoing cataract surgery to be within $+/-1.0 \mathrm{D}$ of predicted refractive outcome. ${ }^{5}$ This was used as our standard for refractive outcome.

We defined early glaucomatous damage as when the visual field MD was less than 6 decibels $(\mathrm{dB}) .^{2}$ Moderate or advanced glaucoma cases were defined as MD of $6 \mathrm{~dB}$ or greater. 
Surgical details

Biometry was performed with the IOL Master (Carl Zeiss Meditex, Jena, Germany); all patients were targeted to achieve emmetropia. The IOL power was calculated using the SRK/T formula with a standardised A constant, except in axial lengths less than $22 \mathrm{~mm}$ in which case the Hoffer $\mathrm{Q}$ formula was used. ${ }^{6}$

All procedures were performed by experienced glaucoma surgeons at the Western Eye Hospital. The procedure was performed under sub-Tenon's or peribulbar local anaesthesia.

Standard cataract surgery was performed with phacoemulsification (Infinity Vision System; Alcon Laboratories, Fort Worth, TX, USA) using a $2.8 \mathrm{~mm}$ temporal clear corneal incision (temporal approach was the surgeon's preference), 1 to 2 side-ports and with further incisions as required to facilitate 360-degree ECP treatment. Foldable acrylic intra-ocular lenses were used, either HOYA PC-60AD or Rayner toric T-flex. The IOL was inserted in the capsular bag. Using the 20G ECP probe (E2; Endo Optiks, Little Silver, NJ, USA), 360-degrees photocoagulation was performed under direct visualization from the base to the tip of each ciliary process, until visible shrinkage and blanching occurred. The treatment energy setting was 0.3 to 0.8 Watts (titrated under direct visualisation of response) with the treatment duration set to continuous. Intracameral cefuroxime $1.0 \mathrm{mg} /$ $0.1 \mathrm{ml}$ and subconjunctival dexamethasone $3.3 \mathrm{mg} / 1 \mathrm{ml}$ injection (Hospira, Lake Forest, California, USA) was given at the end of surgery. A standard post-operative regime of 2 hourly dexamethasone and 6 hourly chloramphenicol drops were given over a 4-6 week period titrated according to post-operative inflammatory response. Patients were instructed to continue initially with their usual glaucoma agents. All patients were reviewed by week 1 and then according to the clinical need, typically twice more in the first 6 weeks. The number of glaucoma agents was reduced if IOP, visual field progress and optic disc assessment remained satisfactory. 
Statistical analysis

Data are presented as mean \pm standard deviation (SD) and frequency (\%). The comparison between the target refraction and the actual post-operative refraction was made using paired t-test. The comparison between the phaco-ECP group and the control group was made using unpaired t-test. Statistical analysis was performed using R software (version 3.3.1). A p-value of less than 0.05 was considered statistically significant. Normality of distribution was tested with Shapiro-Wilk normality test. 


\section{Results}

Sixty-two eyes (10 patients had bilateral surgery) out of the 103 eyes, which underwent phaco-ECP at a single tertiary referral center (The Western Eye Hospital, London, UK) between January 2006 and August 2012, were included in the study with 62 eyes of 62 patients who had phacoemulsification alone as the control group. The mean age for the phaco-ECP group and the control group was $76(\mathrm{SD} \pm 12)$ years and $74(\mathrm{SD} \pm 11)$ years, respectively. In the phaco-ECP group, $58.3 \%$ of the patients were male and in the control group $37 \%$ of the patients were male. The mean follow-up period for all patients was 21 months (range 2 week to 6 year 2 months). The mean visual field MD was $-17.01 \mathrm{~dB}$ (ranging from $-2.44 \mathrm{~dB}$ to $-30.2 \mathrm{~dB}$ ) for the phaco-ECP group. The pre-operative number of glaucoma agents was $2.7 \pm 0.9$ for the phaco-ECP group. Fifteen patients $(27.8 \%)$ in the phaco-ECP group had previous glaucoma procedures including 13 patients who underwent trabeculectomy with or without mitomycin C (MMC), 1 Argon laser trabeculoplasty and 1 transcleral cyclodiode laser. The reasons for exclusions for phaco-ECP group are listed in Table 1.

The majority of patients, 59 eyes $(95.2 \%)$ in the phaco-ECP group and 61 eyes $(98.4 \%)$ in phacoemulsification group, either improved or maintained their visual acuity (Table 2). More than three quarters of the eyes in the phaco-ECP group $(75.8 \%, \mathrm{n}=47)$ had 2 lines or more improvement in their VA. Three eyes in 2 patients $(4.8 \%)$ experienced worsening of the visual acuity, of 3 to 4 Snellen lines, following the surgery. Of these, one patient who had bilateral phaco-ECP had a history of ocular cicatricial pemphigoid (OCP) with end stage refractory glaucoma. One patient had advanced age-related macular degeneration (AMD).

In the phaco-ECP group, $90.3 \%(n=56)$ of all eyes achieved a refractive outcome within $+/-1 \mathrm{D}$ of the target spherical equivalent refraction, whereas in the control group, $100 \%(n=62)$ of eyes achieved this (Figure 1). The difference between the groups is not statistically significant. The refractive outcome of both groups satisfies the standard established by the Royal College of Ophthalmology 2010 guidelines, which states that at least $85 \%$ of patients should achieve the refractive outcome within +/-1D of the target spherical equivalent refraction. ${ }^{7}$ However, when considering the number of cases that achieved the refractive outcome within $+/-0.5 \mathrm{D}$ of the target spherical equivalent refraction in each group, there was a statistically significant difference. Less than $50 \%$ 
of cases $(48.4 \%)$ in the phaco-ECP group achieved the refractive outcome within $+/-0.5 \mathrm{D}$ of the target spherical equivalent refraction whereas $100 \%$ of cases achieved this in the control group (table 3 ). The mean difference between the target and the actual post-operative refraction was $-0.05 \mathrm{D}(\mathrm{SD} \pm 0.7 \mathrm{D})$ in the phaco-ECP group and was 0.09D ( $\mathrm{SD} \pm 0.2 \mathrm{D})$ in the control group. In all three eyes where Rayner T-Flex toric lenses were used the post-operative refraction within +/-0.5D of the target refraction was achieved (range from -0.25D to 0.375D).

Subgroup analysis of the 6 eyes, which were outside the targeted +/-1D refractive outcome in the phaco-ECP group, demonstrates the mean difference of $-1.2 \mathrm{D}(\mathrm{SD} \pm 1.68)$ between the target and the actual refraction. The majority of these eyes, $83.3 \%(n=5)$, displayed myopic shift with post-operative SE ranging between $-1.19 \mathrm{D}$ and $-2.72 \mathrm{D}$. Only one eye (16.7\%) had hypermetropic shift with a SE of +1.57D. Co-morbidities and potential causes for refractive surprise were studied (Table 4). One eye developed fibrinous uveitis and was treated with intensive topical steroid. Five eyes had moderate to advanced field defect with mean MD -19.2dB (range from 11.5dB to $-29.8 \mathrm{~dB}$ ). Three eyes had previous trabeculectomy and the other 3 eyes had co-existing age-related macular degeneration.

In 54 eyes in the phaco-ECP group, the data on IOP, number of glaucoma agents used and history of previous surgery were obtained. Pre-operative mean IOP was $20.4 \pm 6.25 \mathrm{mmHg}$ and the post-operative IOP mean was $14.4 \pm 3.95 \mathrm{mmHg}$. There was an average of $6.0 \mathrm{mmHg}$, or $29.4 \%$ reduction of IOP. Fifty-three eyes $(98.1 \%)$ had post-operative IOP equal to or less than $21 \mathrm{mmHg}$. Seven eyes (13.0\%) achieved this without any topical medication post-operatively. There was an average $29.6 \%$ reduction of number of glaucoma agents (from 2.7 to 1.9). Both the reduction in IOP and the number of glaucoma agents were statistically significant (Table 5).

Complications

Four eyes $(6.5 \%)$ in the phaco-ECP group developed marked post-operative uveitis and required a more intensive post-operative topical steroid regime. All of these eyes had VA of 6/6 at their last follow-up. One eye $(1.6 \%)$ developed fibrinous uveitis with a pupillary membrane and required YAG laser. One eye with advanced 
glaucoma required intracameral tissue plasminogen activator injection with synechiolysis 1 month after phacoECP. The BCVA of hand movement (HM) remained unchanged but this was not unexpected. One patient, with pre-existing OCP and bilateral juxtafoveal telangiectasia, developed post-operative macular oedema. There were no cases of hypotony, lens subluxation or dislocation and there was no case that required a capsular tension ring. No complication occurred in the phacoemulsification group. 


\section{Discussion}

In this study, we demonstrated that the refractive outcome of phaco-ECP is comparable to the control group who underwent phacoemulsification alone with the majority of eyes, $90.3 \%$ achieving within +/-1D of the target refraction using current validated formulae for biometry. This meets the Royal College of Ophthalmology guideline for cataract surgery. ${ }^{5}$ Also almost half of our refractive outcome for the eyes in the phaco-ECP group was within $0.5 \mathrm{D}$ of the target; this is similar to the $55 \%$ reported in previous phacoemulsification-only study. ${ }^{7}$ In our series, all of the eyes in the phacoemulsification only group achieved within $0.5 \mathrm{D}$ of the target refraction.

Five of the 6 patients who were not within +/-1D of the target refraction in the phaco-ECP group experienced myopic shift. This may be explained by possible anterior displacement of the intraocular lens caused by zonular contraction following cyclophotocoagulation. The average age of the patients who did not achieve the target refractive outcome was older $81.5 \pm 11.5$ years compared to the patients who achieved the target refractive outcome, $76 \pm 7.9$ years even though the difference was not statistically significant $(\mathrm{p}=0.61)$. Zonules may become weaker due to age and this could contribute to the higher degrees of zonular movement when cyclophotocoagulation energy was applied to the adjoining ciliary processes, which in turn causes anterior displacement of the intraocular lens, thus myopic shifts. Another possible explanation is the difference in the power of cyclophotocoagulation used during phaco-ECP. However as the end-point is the whitening and shortening of ciliary process, which is observed under visualization, it is unlikely over- or under-treatment occurred.

Our group chose to treat 360-degrees of ciliary processes, as we believe it facilitates maximum IOP reduction. Other groups have also reported this as their preferred treatment protocol. ${ }^{8}$ Ciliary body rotation via external indentation, as advocated by some in order to optimise access to additional posterior rows of ciliary processes and to treat the maximum possible surface area of each ciliary process, was not performed ${ }^{9}$ There were no cases of hypotony. 
Regarding our visual acuity outcome, $4.8 \%(n=3)$ experienced worsening of the visual acuity following phacoECP. All three eyes had co-existing macular pathologies including bilateral ocular cicatricial pemphigoid and post -operative macular oedema in two eyes and one eye with age-related macular oedema. This could be due to the guarded prognosis in patients with advanced glaucoma due to impaired optic disc function and coexisting macular pathologies in both eyes. Also these patients who have been treated with multiple topical glaucoma agents are likely to have suffered from corneal epithelial toxicity, which can compromise the visual outcome. It has been also noted that $50 \%$ of our cohort had visual acuity of $6 / 9$ or better pre-operatively. This may produce a ceiling effect hence failing to show further VA improvement following phaco-ECP. These factors may explain why our VA outcome may differ from a previous report by other group, ${ }^{2}$ where no patients lost BCVA as they only included the early glaucoma populations.

Limitations of the study

The main limitation of the study is that it is retrospective. A larger prospective series will be useful to determine whether there are any characteristics of the patients who are more likely to be refractive surprises. In addition, our post-operative refraction was obtained at various periods after the procedure and was obtained by 2 different methods (subjective and autorefraction). The change of post-operative refractive SE over time has only been studied on standard phacoemulsification cases. It demonstrated that there was no significant difference among the values at 1 week, 1 month, and 4 months. ${ }^{10,11}$ However such data does not exist with phaco-ECP and it is possible that the refractive error may not have stabilised and continues to change over time when our refraction readings were taken. Also, other parameters maybe useful in future studies to investigate causes of visual loss and refractive surprise, such as pre- and post-operative macular OCT and corneal endothelial cell count. 


\section{Conclusions}

Refractive outcome after phaco-ECP is comparable to phacoemulsification alone. This study suggests that the refractive outcome may be more variable when the eye undergoes phaco-ECP rather than phacoemulsification alone. However, the majority of patients will achieve the refractive outcome within 1D of the target spherical equivalent. Therefore, the intraocular lens power can be selected as for phacoemulsification alone. The authors recommend that no modification of biometry formulae is necessary for the eyes that undergo phaco-ECP.

Phaco-ECP should be considered as an effective, safe and predictable surgical treatment option for glaucoma patients with co-existing cataract. 


\section{Figure Captions}

Fig. 1 Variance of the difference between the target and the actual spherical equivalent refraction in dioptres for the phaco-ECP group and the phacoemulsification only control group. 


\section{References}

1. Musch DC, Gillespie BW, Niziol LM, Janz NK, Wren PA, Rockwood EJ (2006) Cataract extraction in the collaborative initial glaucoma treatment study: incidence, risk factors, and the effect of cataract progression and extraction on clinical and quality-of-life outcomes. Collaborative Initial Glaucoma Treatment Study Group. Arch Ophthalmol 124(12): 169-700

2. Lindfield D, Ritchie RW, Griffiths MF (2012) 'Phaco-ECP': combined endoscopic cyclophotocoagulation and cataract surgery to augment medical control of glaucoma. BMJ Open 30: 2(3)

3. Clement CI, Kampougeris G, Ahmed F, Cordeiro MF, Bloom PA (2013) Combining phacoemulsification with endoscopic cyclophotocoagulation to manage cataract and glaucoma. Clin Experiment Ophthalmol 41(6): 546-51

4. Olsen T (2007) Calculation of intraocular lens power: a review. Acta Ophthalmologica Scandinavica 85(5): 472-85

5. The Royal College of ophthalmologists. Cataract Surgery Guideline September 2010. [Online]. Available from: http://www.rcophth.ac.uk/

6. Aristodemou P, Knox Cartwright NE, Sparrow JM, Johnston RL (2011) Formula choice: Hoffer Q, Holladay 1, or SRK/T and refractive outcomes in 8108 eyes after cataract surgery with biometry by partial coherence interferometry. J Cataract Refract Surg 37(1): 63-71

7. Gale RP, Saldana M, Johnston RL, Zuberbuhler B, McKibbin M (2009) Benchmark standards for refractive outcomes after NHS cataract surgery. Eye (London) 23(1): 149-52 
8. Francis BA, Kawji AS, Vo NT, Dustin L, Chopra V (2011) Endoscopic cyclophotocoagulation (ECP) in the management of uncontrolled glaucoma with prior aqueous tube shunt. J Glaucoma 20 (8), 523527

9. Lima FE, Magacho L, Carvalho DM, Susanna R Jr, Avila MP (2004) A prospective, comparative study between endoscopic cyclophotocoagulation and the Ahmed drainage implant in refractory glaucoma. $\mathbf{J}$ Glaucoma 13(3): 233-7

10. Lake D, Fong K, Wilson R (2005). Early refractive stabilization after temporal phacoemulsification: what is the optimum time for spectacle prescription? Journal of Cataract \& Refractive Surgery 31(9):1845

11. De Juan V, Herreras JM, Pérez I, Morejón Á, Río-Cristóbal A, Martín R, Fernandez I, Rodriguez G (2013) Refractive stabilization and corneal swelling after cataract surgery. Optom Vis Sci. 90(1): 31-6 